\title{
Gangrenous Adult Midgut Volvulus from Midgut Malrotation: A Case Report
}

\author{
${ }^{1}$ Uwaezuoke S. C., ${ }^{2}$ Udoye E. P. \\ M.B.,BS, FWACS, M.B.,BS, B.MED SC, FMCPath. \\ ${ }^{1}$ Department of Surgery, Federal Medical Centre, Yenagoa, Bayelsa State, Nigeria. \\ ${ }^{2}$ Department of Anatomical Pathology. Niger Delta University Teaching Hospital, Okolobiri, Bayelsa State, \\ Nigeria.
}

\begin{abstract}
Malrotation of the midgut is mainly a childhood congenital anomaly. Adult Midgut malrotation is rare with the clinical symptomatology varied and non-specific making pre-operative diagnosis difficult. Midgut volvulus from malrotation is a true surgical emergency requiring early intervention to prevent bowel necrosis and death. We report a $27 y \mathrm{r}$ old male who was referred to our centre with paralytic ileus post appendectomy. He underwent emergency laparotomy with findings of gangrenous volvulus involving almost the entire midgut from malrotation. He had bowel resection with jejunocolic anastomosis. Post-operatively he was referred on account of short bowel.A high index of suspicion is needed in acute abdomen with atypical features to allow for early diagnosis and prompt intervention to avert this catastrophic event.
\end{abstract}

Keywords: Adult, midgut malrotation, volvulus, gangrenous

\section{Introduction}

Midgut malrotation is thought to affect approximately 1 in 500 live births and about $80 \%$ of patients with malrotation will present in the first month of life, most within the first week. ${ }^{1 \text { \&2 }}$

Adult midgut malrotation is very rare and its incidence has been reported to be between $0.2 \%$ and $0.5 \%$. ${ }^{3,4 \& 5}$

We herein report a case of an adut male with gangrenous midgut volvulus from midgut malrotation first diagnosed as acute appendicitis, highlighting the need for adequate, appropriate clinical evaluation and intervention in a resource poor environment to avoid this catastrophic event.

\section{Case Report}

A 27 year old man was referred to the federal medical centre from a private health facility on account of paralytic ileus 2 days post appendectomy.

He had had lower abdominal pains for 2 days the year before and was managed conservatively for suspected appendicitis.

He went into shock on the first day post appendectomy and with a packed cell volume (PCV) of $21 \%$ was transfused two units of blood. He developed progressive abdominal distension with vomiting on the second day post appendectomy and was subsequently referred to our hospital.

Upon presentation has was pale with pulse rate of 136beats/minute, respiratory rate 36 cycles/minute, temperature $37.5^{\circ} \mathrm{C}$ and blood pressure $140 / 90 \mathrm{mmHg}$. His abdomen was grossly distended with an oblique right iliac fossa incision wound. There was generalized tenderness with absent bowel sounds. A repeat PCV was $20 \%$, serum electrolytes and urea creatinine values were within normal limits. There was marked microscopic haematuria on urine analysis. Chest x-ray had bilateral pneumonic changes but no air under the diaphragm. Plain abdominal X-ray showed generalized haziness in the abdominal cavity, dilated stomach and colon loop with gas in the rectum. Abdominopelvic scan suggested generalized dilated bowel loops with minimal ascities. Computerized tomography scan examination was unavailable. He was resuscitated with two units of blood and based on a tentative diagnosis of peritonitis from post-operative haemoperitoneum (possible a slipped ligature), he had emergency exploratory laparotomy. 


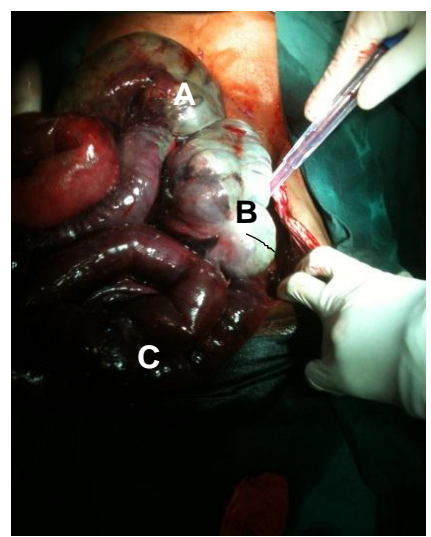

Figure 1: Gangrenous (A)Transverse colon, (B)Caecum(on the left side), (C)Small bowel.

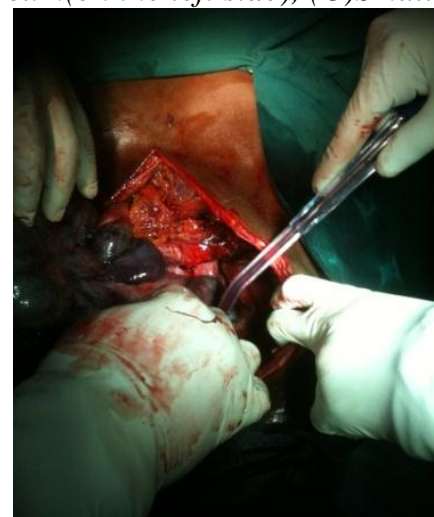

Figure 3: Showing the stomach above the distal transverse colon, after retraction of the gangrenous midgut volvulus to the right.

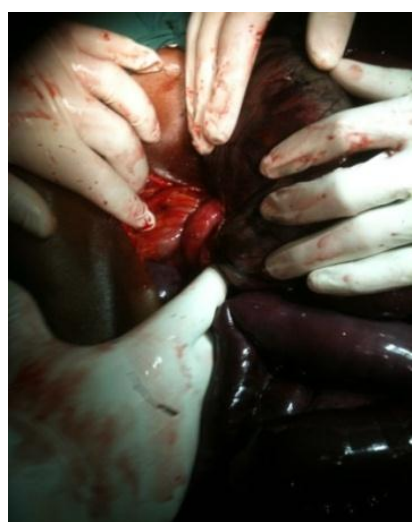

Figure 2: Volvulus after first derotation.

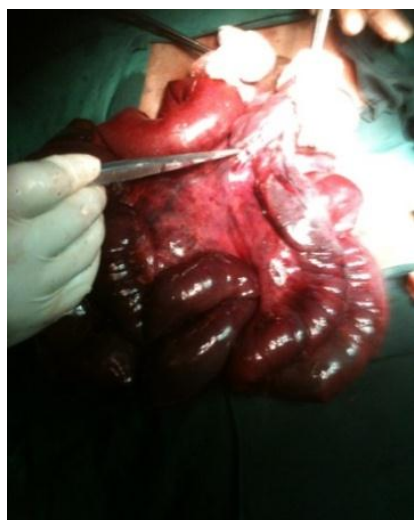

Figure 4: Showing appendix stump with small bowel entering the caecum from the right after decompression and derotation.

Operative findings were a litre of dark haemorrhagic fluid, gangrenous small bowel, caecum, ascending colon, and proximal half of transverse colon, all on a single elongated mobile mesentery completely peritonised with a narrow base. The midgut was involved in a volvulus at the level of the proximal jejunum and mid transverse colon with two complete clockwise turns sparing the first $10 \mathrm{~cm}$ of the viable jejunum. Appendix stump was noted. After decompressing the bowel loops and untwisting in a counter clockwise direction, the small bowel laid on the right side entering the caecum from the right at the upper left abdomen. Adhesions were divided. The gangrenous bowel was resected with primary jejuno-colic anastomosis.

Histopathological evaluation of the resected bowel showed pan-haemorrhagic necrosis with marked congestion of mesenteric blood vessels and lymphatics. No other pathology was identified and the resection margins were viable.

Postoperatively he was transfused three units of blood. He was commenced on oral rehydration solution in addition to his intravenous fluids. He developed surgical site infection postoperatively which resolved on conservative management. He was referred out on account of his short bowel for specialist care.

\section{Discussion}

Intestinal malrotation generally means any deviation from the normal $270^{\circ}$ counter clockwise rotation from the midgut during embryological development. Normal intrauterine intestinal rotation primarily involves the midgut and it is divided into 3 stages.

Stage 1 involves extrusion of the midgut into the extra embryonic cavity, a $90{ }^{0}$ counter clockwise rotation and return of the midgut into the fetal abdomen.

The $2^{\text {nd }}$ stage involves another counterclockwise rotation within the abdominal cavity to complete a $270{ }^{0}$ rotation. This places the duodenal " $\mathrm{C}$ " loop behind the superior mesenteric artery with the ascending colon to the right, the transverse colon above and the descending colon to the left.

The $3^{\text {rd }}$ stage involves fusion and anchoring of the mesentery. The caecum descends and both the ascending and descending colon get attached to the posterior abdominal wall. Thus stage one (anomalies of migration) includes omphalocoele, stage 2 anomalies (anomalies of rotation) include non-rotation, malrotation, reverse rotation. 
While stage 3 anomalies (anomalies of fixation) include unattached duodenum, mobile caecum, unattached small bowel mesentery, Ladd's bands. ${ }^{5,6 \& 7}$

The classical presentation of malrotation in neonates is bilious vomiting. ${ }^{2 \& 7}$

Many adult patients with midgut malrotation are usually asymptomatic, diagnosis made either on imaging investigations for unrelated conditions or at operations for other pathologies. ${ }^{8 \& 9}$

However midgut malrotation in older child or adults may cause chronic abdominal symptoms such as intermittent colicky abdominal pains, vomiting, diarrhoea or constipation. These sometimes last for years and have been misdiagnosed as psychiatric disorders, Crohn's disease, adhesions or malingering. ${ }^{9 \& 10}$

Midgut malrotation can also present acutely as acute bowel obstruction. In the adult with malrotation midgut volvulus is the most common cause of intestinal obstruction. ${ }^{8} \& 9$

Abdominal radiographs, ultrasound scan, computerised tomography scan (CT), magnetic resonance imaging scan (MRI) have been employed in the diagnosis of midgut malrotation. ${ }^{2,8 \& 11}$

Finding on plain abdominal radiographs in midgut volvulus are usually abnormal, but unspecific. However upper gastrointestinal contrast series(the study of choice in the sick infant or child) shows the diagnostic atypical Corkscrew appearance of the proximal small bowel. ${ }^{2 \& 11}$

In adults with acute symptoms CT is generally performed and the characteristic diagnostic finding of midgut volvulus may be seen. The diagnostic whirl or whirlpool sign shows the swirling appearance of bowel and mesentery twisted around the superior mesenteric artery (SMA) axis. A similar scenario can be seen on ultrasonography ${ }^{9} \& 11$. Our patient had unusual findings on plain abdominal X-ray but they are not specific and abdominal ultrasonography in this case was not helpful.

The treatment of acutely symptomatic patients with midgut volvulus is surgical intervention. ${ }^{6 \& 8}$

Ladd procedure is most commonly done. It consists of four parts which include derotation of the volvulus if present, division of Ladds bands over the duodenum, widening of the narrow roots of the small bowel mesentery by mobilizing the duodenum and appendectomy to prevent future diagnostic dilemma of an abnormally located inflamed appendix. ${ }^{8,9 \& 12}$ Ladd procedure can also be done laparoscopically in patients with malrotation ${ }^{13}$.

Alas for the individual with ischemia and bowel necrosis resection is done as was the case in our patient. A resultant condition from massive small bowel resection in gangrenous midgut volvulus is short bowel syndrome. This often requires a multidisciplinary approach with total parenteral nutrition. The challenges involved are quite daunting in the face of poor finance, unavailability of materials and personnel lack. ${ }^{7 \& 14}$

\section{Conclusion}

Midgut malrotation though rare should be considered in managing an adult with recurrent unspecific abdominal pains. Clinicians and radiologists should have a high index of suspicion when managing acute abdominal cases with atypical features with early CT and or abdominal ultrasonography to exclude midgut malrotation and volvulus. When in doubt with limited facilities, early exploratory laparotomy still remains the most logical option to exclude or curtail this potential vascular disaster.

\section{References}

[1]. Tores AM, Ziegler MM; Malrotation of the intestine. World J Surg 1993, 17:326-331

[2]. Strouse PJ. Disorders of intestinal rotation and fixation("malrotation"). PediatrRadiol 2004; 34 : 837 - 851.

[3]. Papaziogas B, Patsas A, Paraskevas G, Tsiaousis P, Koutelidakis I, Christoforakis C, Atmatzidis K . Adult Intestinal Malrotation: A Case Report. The Internet Journal of Surgery. 2008 volume19 number 2

[4]. Sing S, Das A, Chawla AS, Arya SV, Chaggar J. A rare presentation of midgut malrotation as an acute intestinal obstruction in an adult: Two case reports and literature reviews.Int J Surg Case Rep 2013; 4:72-75(PMID:23123419 DOI:10.1016/j.ijscr.2012.10.005)

[5]. Mallick IH, Igbal R, Davis JB. Situs inversus abdominis and malrotation in an adult with Ladd's band formation leading to intestinal ischaemia. World J Gastroenterol 2006;12:4093-5.

[6]. Gohl ML, DeMeesterTR. Midgut malrotation in adults: an aggressive approach. Am J Surg 1975; 129:319-323.

[7]. Askegard-Giesmann JR, Amah CC, Kenny BD. Intestinal Malrotation and midgut volvulus. In: Ameh E, Bickler S, Lakhoo K, Nwomeh B, Poenaru D, editors. Paediatric Surgery: A Comprehensive Textbook for Africa,- ed. Seattle: Global- HELP Organization; 2010:p393-396.

[8]. Emanuwa OF, Ayantunde AA, Davis TW. Midgut malrotation first presenting as acute bowel obstruction in adulthood: A case report and literature review. World J Emerg Surg 2011; 6:22

[9]. Bernstein SM, Russ PD. Midgut volvulus: a rare cause of acute abdomen in an adult patient. AJR 1998; 171:639-641.

[10]. Devlin HB. Midgut malrotation causing intestinal obstruction in adult patients. Ann Roy. Coll. Surg Engl 1971, 48:227-237.

[11]. Pickhardt PJ, Bhalla S: Intestinal malrotation in adolescents and adults: Spectrum of clinical and imaging features. American Journal of Radiology 2002, 179: 1429-1435.

[12]. Ladd WE: Surgical diseases of the alimentary tract in infants. N Engl J Med 1936, 215:705-708.

[13]. Matzke GM, Dozois EJ, Larson DW, Moir CR. Surgical management of intestinal malrotation in adults: comparative results for open laparoscopic Ladd procedures. Surg Endosc 2005; 19:1416-1419.

[14]. Wallberg SV,Qvist N. Increased risk of complications in acute onset intestinal rotation. DAN MED J 2013; $60(12)$ : A4744. 